\title{
An Experimental Mathematics Approach to the Area Statistic of Parking Functions
}

Yukun YAO and Doron ZEILBERGER

\begin{abstract}
We illustrate the experimental, empirical, approach to mathematics (that contrary to popular belief, is often rigorous), by using parking functions and their 'area' statistic, as a case study. Our methods are purely finitistic and elementary, taking full advantage, of course, of our beloved silicon servants.
\end{abstract}

\section{Accompanying Maple package and input and output files}

This article is accompanied by a Maple package ParkingStatistics.txt available from the front of this article

http://sites.math.rutgers.edu/ zeilberg/mamarim/mamarimhtml/par.html, ,

where readers can also find lots of output files, and nice pictures.

Pre-History (and Pre PC)

Once upon a time, way back in the nineteen-sixties, there was a one-way street (with no passing allowed), with $n$ parking spaces bordering the sidewalk. Entering the street were $n$ cars, each driven by a loyal husband, and sitting next to him, dozing off, was his capricious (and a little bossy) wife. At a random time (while still along the street), the wife wakes up and orders her husband, park here, darling!. If that space is unoccupied, the hubby gladly obliges, and if the parking space is occupied, he parks, if possible, at the first still-empty parking space. Alas, if all the latter parking spaces are occupied, he has to go around the block, and drive back to the beginning of this one-way street, and then look for the first available spot. Due to construction, this wastes half an hour, making the wife very cranky.

Q: What is the probability that no one has to go around the block?

$\mathbf{A}:(n+1)^{n-1} / n^{n} \asymp \frac{e}{n+1}$.

Both the question and its elegant answer are due to Alan Konheim and Benji Weiss [KW].

\section{Parking Functions}

Suppose wife $i(1 \leq i \leq n)$ prefers parking-space $p_{i}$, then the preferences of the wives can be summarized as an array $\left(p_{1}, \ldots, p_{n}\right)$, where $1 \leq p_{i} \leq n$. So altogether there are $n^{n}$ possible preference-vectors, starting from $(1, \ldots, 1)$ where it is clearly possible for everyone to park, and ending with $(n, \ldots, n)$ (all $n$ ), where every wife prefers the last parking space, and of course it is impossible. Given a preference vector $\left(p_{1}, \ldots, p_{n}\right)$, let $\left(p_{(1)}, \ldots, p_{(n)}\right)$ be its sorted version, arranged in (weakly) increasing order. 
For example if $\left(p_{1}, p_{2}, p_{3}, p_{4}\right)=(3,1,1,4)$ then $\left(p_{(1)}, p_{(2)}, p_{(3)}, p_{(4)}\right)=(1,1,3,4)$.

We invite our readers to convince themselves that a parking-space preference vector $\left(p_{1}, \ldots, p_{n}\right)$ makes it possible for every husband to park without inconveniencing his wife if and only if $p_{(i)} \leq i$ for $1 \leq i \leq n$. This naturally leads to the following definition.

Definition of a Parking Function: A vector of positive integers $\left(p_{1}, \ldots, p_{n}\right)$ with $1 \leq p_{i} \leq n$ is a parking function if its (non-decreasing) sorted version $\left(p_{(1)}, \ldots, p_{(n)}\right)$ (i.e. $p_{(1)} \leq p_{(2)} \leq \ldots \leq$ $p_{(n)}$, and the latter is a permutation of the former) satisfies

$$
p_{(i)} \leq i \quad, \quad(1 \leq i \leq n) \quad .
$$

As we have already mentioned above, Alan Konheim and Benji Weiss ([KW]) were the first to state and prove the following theorem.

The Parking Function Enumeration Theorem: There are $(n+1)^{n-1}$ parking functions of length $n$.

There are many proofs of this lovely theorem, possibly the slickest is due to the brilliant human Henry Pollak, (who apparently did not deem it worthy of publication. It is quoted, e.g. in [FR]). It is nicely described on pp. 4-5 of [St1] (see also [St2]), hence we will not repeat it here. Instead, as a warm-up to the 'statistical' part, and to illustrate the power of experiments, we will give a much uglier proof, that, however, is motivated.

Before going on to present our (very possibly not new) 'humble' proof, we should mention that one natural way to prove the Konheim-Weiss theorem is by a bijection with labeled trees on $n+1$ vertices, that Arthur Cayley famously proved is also enumerated by $(n+1)^{n-1}$. The first such bijection, as far as we know, was given by the great formal linguist, Marco Schützenberger ([Sc]). This was followed by an elegant bijection by the classical combinatorial giants Dominique Foata and John Riordan [FR], and others.

Since we know (at least!) 16 different proofs of Cayley's formula (see, e.g. [Z3]), and at least four different bijections between parking functions and labeled trees, there are at least 64 different proofs (see also [St3], ex. 5.49) of the Parking Enumeration theorem. To these one must add proofs like Pollak's, and a few other ones.

Curiously, our 'new' proof has some resemblance to the very first one in [KW], since they both use recurrences (one of the greatest tools in the experimental mathematician's tool kit!), but our proof is (i) motivated (ii) experimental (yet fully rigorous).

\section{An Experimental Mathematics Motivated Proof of the Kohnheim-Weiss Parking Enu- meration Theorem}

When encountering a new combinatorial family, the first task is to write a computer program to enumerate as many terms as possible, and hope to conjecture a nice formula. One can also try and 
"cheat" and use the great OEIS, to see whether anyone came up with this sequence before, and see whether this new combinatorial family is mentioned there.

A very brute force approach, that will not go very far (but would suffice to get the first five terms needed for the OEIS) is to list the superset, in this case all the $n^{n}$ vectors in $\{1 \ldots n\}^{n}$ and for each of them sort it, and see whether the condition $p_{(i)} \leq i$ holds for all $1 \leq i \leq n$. Then count the vectors that pass this test.

But a much better way is to use dynamical programming to express the desired sequence, let's call it $a(n)$, in terms of values $a(i)$ for $i<n$.

Let's analyze the anatomy of a typical parking function of length $n$. A natural parameter is the number of 1's that show up, let's call it $k(0 \leq k \leq n)$. i.e.

$$
p_{(1)}=1 \quad, \quad \ldots \quad, \quad p_{(k)}=1 \quad, \quad 2 \leq p_{(k+1)} \leq k+1 \quad, \quad \ldots \quad, \quad p_{(n)} \leq n \quad .
$$

Removing the 1's yields a shorter weakly-increasing vector

$$
2 \leq p_{(k+1)} \leq p_{(k+2)} \leq \cdots \quad \leq p_{(n)},
$$

satisfying

$$
p_{(k+1)} \leq k+1 \quad, \quad p_{(k+2)} \leq k+2 \quad, \quad \cdots \quad, \quad p_{(n)} \leq n
$$

Define

$$
\left(q_{1}, \ldots, q_{n-k}\right):=\left(p_{(k+1)}-1, \ldots, p_{(n)}-1\right) .
$$

The vector $\left(q_{1}, \ldots, q_{n-k}\right)$ satisfies

$$
1 \leq q_{1} \leq \ldots \leq q_{n-k}
$$

and

$$
q_{1} \leq k \quad, \quad q_{2} \leq k+1 \quad, \quad \ldots \quad, \quad q_{n-k} \leq n-1 \quad .
$$

We see that the set of parking functions with exactly $k$ 1's may be obtained by taking the above set of vectors of length $n-k$, adding 1 to each component, scrambling it in everywhich way, and inserting the $k$ 1's in everywhich way.

Alas, the 'scrambling' of the set of such $q$-vectors is not of the original form. We are forced to consider a more general object, namely scramblings of vectors of the form $p_{(1)} \leq \ldots \leq p_{(n)}$ with the condition

$$
p_{(1)} \leq a \quad, \quad p_{(2)} \leq a+1 \quad, \quad \cdots \quad, \quad p_{(n)} \leq a+n-1 \quad,
$$

for a general, positive integer $a$, not just for $a=1$. So in order to get the dynamical programming recurrence rolling we are forced to introduce a more general object, called an $a$-parking function. This leads to the following definition. 
Definition of an a-Parking Function: A vector of positive integers $\left(p_{1}, \ldots, p_{n}\right)$ with $1 \leq p_{i} \leq$ $n+a-1$ is an $a$-parking function if its (non-decreasing) sorted version $\left(p_{(1)}, \ldots, p_{(n)}\right)$ (i.e. $p_{(1)} \leq p_{(2)} \leq \ldots \leq p_{(n)}$, and the latter is a permutation of the former) satisfies

$$
p_{(i)} \leq a+i-1 \quad, \quad(1 \leq i \leq n) \quad .
$$

Note that the usual parking functions are the special case $a=1$. So if we would be able to find an efficient recurrence for counting $a$-parking functions, we would be able to answer our original question.

So let's redo the above 'anatomy' for these more general creatures, and hope that the two parameters $n$ and $a$ would suffice to establish a recursive scheme, and we won't need to introduce yet more general creatures.

Let's analyze the anatomy of a typical $a$-parking function of length $n$. Again, a natural parameter is the number of 1 's that show up, let's call it $k(0 \leq k \leq n)$. i.e.

$$
p_{(1)}=1 \quad, \quad \ldots \quad, \quad p_{(k)}=1 \quad, \quad 2 \leq p_{(k+1)} \leq a+k \quad, \quad \ldots \quad p_{(n)} \leq a+n-1 .
$$

Removing the 1-s yields a sorted vector

$$
2 \leq p_{(k+1)} \leq p_{(k+2)} \leq \cdots \leq p_{(n)}
$$

satisfying

$$
p_{(k+1)} \leq k+a \quad, \quad p_{(k+2)} \leq k+a+1 \quad, \quad \ldots \quad, \quad p_{(n)} \leq n+a-1
$$

Define

$$
\left(q_{1}, \ldots, q_{n-k}\right):=\left(p_{(k+1)}-1 \quad, \quad \ldots \quad, \quad p_{(n)}-1\right) .
$$

The vector $\left(q_{1}, \ldots, q_{n-k}\right)$ satisfies

$$
q_{1} \leq \ldots \leq q_{n-k}
$$

and

$$
q_{1} \leq k+a-1 \quad, \quad q_{2} \leq k+a \quad, \quad \ldots \quad, \quad q_{n-k} \leq n+a-1 \quad .
$$

We see that the set of $a$-parking functions with exactly $k 1$ 's may be obtained by taking the above set of vectors of length $n-k$, adding 1 to each component, scrambling it in everywhich way, and inserting the $k$ 1's in everywhich way.

But now the set of scramblings of the vectors $\left(q_{1}, \ldots q_{n-k}\right)$ is an old friend!. It is the set of $(a+k-1)$-parking functions of length $n-k$. To get all $a$-parking functions of length $n$ with exactly $k$ ones we need to take each and every member of the set of $(a+k-1)$-parking functions of length $n-k$, add 1 to each component, and insert $k$ ones in every which way. There are $\left(\begin{array}{l}n \\ k\end{array}\right)$ ways of doing it. Hence the number of $a$-parking functions of length $n$ with exactly $k$ ones is $\left(\begin{array}{l}n \\ k\end{array}\right)$ times the 
number of $(a+k-1)$-parking functions of length $n-k$. Summing over all $k$ between 0 and $n$ we get the following recurrence.

\section{Fundamental Recurrence for $a$-parking functions}

Let $p(n, a)$ be the number of $a$-parking functions of length $n$. We have the recurrence

$$
p(n, a)=\sum_{k=0}^{n}\left(\begin{array}{l}
n \\
k
\end{array}\right) p(n-k, a+k-1),
$$

(FundamentalRecurrence)

subject to the boundary conditions $p(n, 0)=0$ for $n \geq 1$, and $p(0, a)=1$ for $a \geq 0$.

Note that in the sense of Wilf [W], this already answers the enumeration problem to compute $p(n, a)$ and hence $p(n, 1)=p(n)$, since this gives us a polynomial time algorithm to compute $p(n)$ (and $p(n, a)$ ).

Moving the term $k=0$ from the right to the left, and denoting $p(n, a)$ by $p_{n}(a)$ we have

$$
p_{n}(a)-p_{n}(a-1)=\sum_{k=1}^{n}\left(\begin{array}{l}
n \\
k
\end{array}\right) p_{n-k}(a+k-1) \text {. }
$$

Hence we can express $p_{n}(a)$ as follows, in terms of $p_{m}(a)$ with $m<n$.

$$
p_{n}(a)=\sum_{b=0}^{a}\left(\sum_{k=1}^{n}\left(\begin{array}{l}
n \\
k
\end{array}\right) p_{n-k}(b+k-1)\right) .
$$

Here is the Maple code that implements it

$\mathrm{p}:=\operatorname{proc}(\mathrm{n}, \mathrm{a})$ local $\mathrm{k}, \mathrm{b}$ :

if $n=0$ then

$\operatorname{RETURN}(1)$

else

factor $(\operatorname{subs}(b=a, \operatorname{sum}(\operatorname{expand}(\operatorname{add}(\operatorname{binomial}(n, k) * \operatorname{subs}(a=a+k-1, p(n-k, a)), k=1 \ldots n)), a=1 \ldots b)))$ :

fi:

end:

If you copy-and-paste this onto a Maple session, as well as the line below,

$[\operatorname{seq}(p(i, a), i=1 \ldots 10)] ;$ 
you would immediately get

$\left[a, a(a+2), a(a+3)^{2}, a(a+4)^{3}, a(a+5)^{4}, a(a+6)^{5}, a(a+7)^{6}, a(a+8)^{7}, a(a+9)^{8}, a(a+10)^{9}\right]$.

Note that these are rigorously proved exact expressions, in terms of general a (i.e. symbolic a) for $p_{n}(a)$, for $1 \leq n \leq 10$, and we can easily get more. The following guess immediately comes to mind

$$
p(n, a)=p_{n}(a)=a(a+n)^{n-1} .
$$

How to prove this rigorously? If you set $q(n, a):=a(a+n)^{n-1}$, since $q(n, 0)=0$ and $q(0, a)=1$, the fact that $p(n, a)=q(n, a)$ would follow by induction once you prove that $q(n, a)$ also satisfies the same fundamental recurrence.

$$
q(n, a)=\sum_{k=0}^{n}\left(\begin{array}{l}
n \\
k
\end{array}\right) q(n-k, a+k-1)
$$

In other words, in order to prove that $p(n, a)=a(n+a)^{n-1}$, we have to prove the identity

$$
a(a+n)^{n-1}=\sum_{k=0}^{n}\left(\begin{array}{l}
n \\
k
\end{array}\right)(a+k-1)(a+n-1)^{n-k-1}
$$

but this is an immediate consequence of the binomial theorem, hence trivial to both humans and machines.

We have just rigorously reproved, via experimental mathematics, the following well-known theorem.

Theorem: The number of $a$-parking functions of length $n$ is

$$
p(n, a)=a(a+n)^{n-1}
$$

In particular, by substituting $a=1$, we reproved the original Konheim-Weiss theorem that $p(n, 1)=$ $(n+1)^{n-1}$.

\section{From Enumeration to Statistics in General}

Often in enumerative combinatorics, the class of interest has natural 'statistics', like height, weight, and IQ for humans, and one is interested rather than, for a finite set $A$,

$$
|A|:=\sum_{a \in A} 1
$$


called the naive counting, and getting a number (obviously a non-negative integer), by the so-called weighted counting,

$$
|A|_{x}:=\sum_{a \in A} x^{f(a)},
$$

where $f:=A \rightarrow Z$ is the statistic in question. To go from the weighted enumeration (a certain Laurent polynomial) to straight enumeration, one sets $x=1$, i.e. $|A|_{1}=|A|$.

Since this is mathematics, and not accounting, the usual scenario is not just one specific set $A$, but a sequence of sets $\left\{A_{n}\right\}_{n=0}^{\infty}$, and then the enumeration problem is to have an efficient description of the numerical sequence $a_{n}:=\left|A_{n}\right|$, ready to be looked-up (or submitted) to the OEIS, and its corresponding sequence of polynomials $P_{n}(x):=\left|A_{n}\right|_{x}$.

It often happens that the statistic $f$, defined on $A_{n}$, has a scaled limiting distribution. In other words, if you draw a histogram of $f$ on $A_{n}$, , and do the obvious scaling, they get closer and closer to a certain continuous curve, as $n$ goes to infinity.

The scaling is as follows. Let $E_{n}(f)$ and $\operatorname{Var}_{n}(f)$ the expectation and variance of the statistic $f$ defined on $A_{n}$, and define the scaled random variable, for $a \in A_{n}$, by

$$
X_{n}(a):=\frac{f(a)-E_{n}(f)}{\sqrt{\operatorname{Var}_{n}(f)}} .
$$

If you draw the histograms of $X_{n}(a)$ for large $n$, they look practically the same, and converge to some continuous limit.

A famous example is coin tossing. If $A_{n}$ is $\{-1,1\}^{n}$, and $f(v)$ is the sum of $v$, then the limiting distribution is the bell shaped curve aka standard normal distribution aka Gaussian distribution.

As explained in [Z4], a purely finitistic approach to finding, and proving, a limiting scaled distribution, is via the method of moments. Using symbolic computation, the computer can rigorously prove exact expressions for as many moments as desired, and often (like in the above case, see [Z4]) find a recurrence for the sequence of moments. This enables one to identify the limits of the scaled moments with the moments of the continuous limit (in the example of coin-tossing [and many other cases], $\frac{e^{-x^{2} / 2}}{\sqrt{2 \pi}}$, whose moments are famously $\left.1,0,1 \cdot 3,0,1 \cdot 3 \cdot 5,0,1 \cdot 3 \cdot 5 \cdot 7,0, \ldots\right)$. Whenever this is the case the discrete family of random variables is called asymptotically normal. Whenever this is not the case, it is interesting and surprising.

\section{The Sum and Area Statistics on $a$-parking functions}

Let $\mathcal{P}(n, a)$ be the set of $a$-parking functions of length $n$.

A natural statistic is the sum

$$
\operatorname{Sum}\left(p_{1}, \ldots, p_{n}\right):=p_{1}+p_{2}+\ldots+p_{n}=\sum_{i=1}^{n} p_{i} .
$$


Another, even more natural (see the beautiful article $[\mathrm{DH}]$ ) happens to be

$$
\operatorname{Area}(p):=\frac{n(2 a+n-1)}{2}-\operatorname{Sum}(p)
$$

Let $P(n, a)(x)$ be the weighted analog of $p(n, a)$, according to Sum, i.e.

$$
P(n, a)(x):=\sum_{p \in \mathcal{P}(n, a)} x^{\operatorname{Sum}(p)}
$$

Analogously, let $Q(n, a)(x)$ be the weighted analog of $p(n, a)$, according to Area, i.e.

$$
Q(n, a)(x):=\sum_{p \in \mathcal{P}(n, a)} x^{\operatorname{Area}(p)}
$$

Clearly, one can easily go from one to the other

$$
Q(n, a)(x)=x^{(2 a+n-1) n / 2} P(n, a)\left(x^{-1}\right) \quad, \quad P(n, a)(x)=x^{(2 a+n-1) n / 2} Q(n, a)\left(x^{-1}\right) .
$$

How do we compute $P(n, a)(x)$ ?, (or equivalently, $Q(n, a)(x)$ ?). It is readily seen that the analog of (FundamentalRecurrence) for the weighted counting is

$$
P(n, a)(x)=x^{n} \sum_{k=0}^{n}\left(\begin{array}{l}
n \\
k
\end{array}\right) P(n-k, a+k-1)(x) \quad, \quad \text { (FundamentalRecurrenceX) }
$$

subject to the initial conditions $P(0, a)(x)=1$ and $P(n, 0)(x)=0$.

So it is almost the same, the "only" change is sticking $x^{n}$ in front of the sum on the right hand side.

Equivalently,

$$
Q(n, a)(x)=\sum_{k=0}^{n}\left(\begin{array}{l}
n \\
k
\end{array}\right) x^{k(k+2 a-3) / 2} Q(n-k, a+k-1)(x) \quad, \quad(\text { FundamentalRecurrenceAreaX) }
$$

subject to the initial conditions $Q(0, a)(x)=1$ and $Q(n, 0)(x)=0$.

Once again, in the sense of Wilf, this is already an answer, but because of the extra variable $x$, one can not go as far as we did before for the naive, merely numeric, counting.

It is very unlikely that there is a "closed form" expression for $P(n, a)(x)$ (and hence $Q(n, a)(x)$ ), but for statistical purposes it would be nice to get "closed form" expressions for

- the expectation, 
- the variance,

- as many factorial moments as possible, from which the 'raw' moments, and latter the centralized moments and finally the scaled moments can be gotten. Then we can take the limits as $n$ goes to infinity, and see if they match the moments of any of the known continuous distributions, and prove rigorously that, at least for that many moments, the conjectured limiting distribution matches.

In our case, the limiting distribution is the intriguing so-called Airy distribution, that Svante Janson prefers to call "area under Brownian excursion". This result was stated and proved in [DH], by using deep and sophisticated continuous probability theory and continuous martingales. Here we will "almost" prove this result, in the sense of showing that the limits of the scaled moments of the area statistic on parking functions coincide with the scaled moments of the Airy distribution up to the 30-th moment, and we can go much further.

But we can do much more than continuous probabilists. We (or rather our computers, running Maple) can find exact polynomial expressions in $n$ and the expectation $E_{1}(n)$. We can do it for any desired number of moments, say 30. Unlike continuous probability theorists, our methods are entirely elementary, only using high school algebra.

We can also do the same thing for the more general $a$-parking functions. Now the expressions are polynomials in $n, a$, and the expectation $E_{1}(n, a)$.

Finally, we believe that our approach, using the recurrence (FundamentalRecurrenceAreaX), can be used to give a full proof (for all moments), by doing it asymptotically, and deriving a recurrence for the leading terms of the asymptotics for the factorial moments that would coincide with the well-known recurrence for the moments of the Airy distribution given, for example in Eqs. (4) and (5) of Svante Janson's article $[\mathrm{J}]$. This is left as a challenge to our readers.

\section{Finding the Expectation}

The expectation of the sum statistic, let's call it $E_{\text {sum }}(n, a)$ is given by (the prime denotes, as usual, differentiation w.r.t. $x$ )

$$
E_{\text {sum }}(n, a)=\frac{P^{\prime}(n, a)(1)}{P(n, a)(1)}=\frac{P^{\prime}(n, a)(1)}{a(a+n)^{n-1}} .
$$

Can we get a closed-form expression for $P^{\prime}(n, a)(1)$, and hence for $E_{\text {sum }}(n, a)$ ?

Differentiating (FundamentalRecurrenceX) with respect to $x$, using the product rule, we get

$$
P(n, a)^{\prime}(x)=x^{n} \sum_{k=0}^{n}\left(\begin{array}{l}
n \\
k
\end{array}\right) P(n-k, a+k-1)^{\prime}(x)+n x^{n-1} \sum_{k=0}^{n}\left(\begin{array}{l}
n \\
k
\end{array}\right) P(n-k, a+k-1)(x) \text {. }
$$

Plugging-in $x=1$ we get that $P(n, a)^{\prime}(1)$, satisfies the recurrence

$$
P(n, a)^{\prime}(1)-\sum_{k=0}^{n}\left(\begin{array}{l}
n \\
k
\end{array}\right) P(n-k, a+k-1)^{\prime}(1)=n \sum_{k=0}^{n}\left(\begin{array}{l}
n \\
k
\end{array}\right) P(n-k, a+k-1)(1)=n p(n, a) .
$$

(FundamentalRecurrenceX1) 
Using this recurrence, we can, just as we did for $p(n, a)$ above, get expressions, as polynomials in $a$, for numeric $1 \leq n \leq 10$, say, and then conjecture that

$$
P^{\prime}(n, a)(1)=\frac{1}{2} a n(a+n-1)(a+n)^{n-1}-\frac{1}{2} \sum_{j=1}^{n}\left(\begin{array}{l}
n \\
j
\end{array}\right) j ! a(a+n)^{n-j} .
$$

To prove it, one plugs in the left side into (FundamentalRecurrenceX1), changes the order of summation, and simplifies. This is rather tedious, but since at the end of the day, these are equivalent to polynomial identities in $n$ and $a$, checking it for sufficiently many special values of $n$ and $a$ would be a rigorous proof.

It follows that

$$
E_{\text {sum }}(n, a)=\frac{n(a+n+1)}{2}-\frac{1}{2} \sum_{j=1}^{n} \frac{n !}{(n-j) !(a+n)^{j-1}} .
$$

This formula first appears in [KY1].

Equivalently,

$$
E_{\text {area }}(n, a)=\frac{n(a-2)}{2}+\frac{1}{2} \sum_{j=1}^{n} \frac{n !}{(n-j) !(a+n)^{j-1}} .
$$

In particular, for the primary object of interest, the case $a=1$, we get

$$
E_{\text {area }}(n, 1)=-\frac{n}{2}+\frac{1}{2} \sum_{j=1}^{n} \frac{n !}{(n-j) !(n+1)^{j-1}} .
$$

This rings a bell! It may written as

$$
E_{\text {area }}(n, 1)=-\frac{n}{2}+\frac{1}{2} W_{n+1}
$$

where $W_{n}$ is the iconic quantity,

$$
W_{n}=\frac{n !}{n^{n-1}} \sum_{k=0}^{n-2} \frac{n^{k}}{k !}
$$

proved by Riordan and Sloane ([RS]) to be the expectation of another very important quantity, the sum of the heights on rooted labeled trees on $n$ vertices. In addition to its considerable mathematical interest, this quantity, $W_{n}$, has great historical significance, it was the first sequence, sequence $A 435$ of the amazing On-Line Encyclopedia of Integer Sequences (OEIS), now with almost 300000 sequences! See [EZ] for details, and far-reaching extensions, analogous to the present paper.

[The reason it is not sequence A1 is that initially the sequences were arranged in lexicographic order.]

Another fact, that will be of great use later in this paper, is that, as noted in [RS], Ramanujan and Watson proved that $W_{n}$ (and hence $W_{n+1}$ ) is asymptotic to

$$
\frac{\sqrt{2 \pi}}{2} n^{3 / 2}
$$


It is very possible that the formula $E_{\text {area }}(n, 1)=-\frac{n}{2}+\frac{1}{2} W_{n+1}$ may also be deduced from the Riordan-Sloane result via one of the numerous known bijections between parking functions and rooted labeled trees. More generally, the results below, for the special case $a=1$, might be deduced, from those of [EZ], but we believe that the present methodology is interesting for its own sake, and besides in our current approach (that uses recurrences rather than the Lagrange Inversion Formula), it is much faster to compute higher moments, hence, going in the other direction, would produce many more moments for the statistic on rooted labeled trees considered in [EZ], provided that there is indeed such a correspondence that sends the area statistic on parking functions (suitably tweaked) to the Riordan-Sloane statistic on rooted labeled trees.

\section{The Limiting Distribution}

Given a combinatorial family, one can easily get an idea of the limiting distribution by taking a large enough $n$, say $n=100$, and generating a large enough number of random objects, say 50000, and drawing a histogram, see Figure 2 in Diaconis and Hicks' insightful article [DH]. But, one does not have to resort to simulation. While it is impractical to consider all $101^{99}$ parking functions of length 100, the generating function $Q(100,1)(x)$ contains the exact count for each conceivable area from 0 to $\left(\begin{array}{c}100 \\ 2\end{array}\right)$. See

http://sites.math.rutgers.edu/ zeilberg/tokhniot/picsParking/Ha100.html ,

for the full histogram.

But an even more informative way to investigate the limiting distribution is to draw the histogram of the probability generating function of the scaled distribution

$$
X_{n}(p):=\frac{\operatorname{Area}(p)-E_{n}}{\sqrt{\operatorname{Var}_{n}}}
$$

where $E_{n}$ and $\operatorname{Var}_{n}$ are the expectation and variance respectively.

See

http://sites.math.rutgers.edu/ zeilberg/tokhniot/picsParking/Da100.html , ,

for $n=100$ and

http://sites.math.rutgers.edu/ zeilberg/tokhniot/picsParking/Da120.html , ,

for $n=120$. They look the same!

As proved in $[\mathrm{DH}]$ (using deep results in continuous probability due to David Aldous, Svante Janson, and Chassaing and Marcket) the limiting distribution is the Airy distribution. We will soon "almost" prove it, but do much more by discovering exact expressions for the first 30 moments, not just their limiting asymptotics. 


\section{Truly Exact Expressions for the Factorial (and hence Centralized Moments)}

In [KY2] there is an "exact" expression for the general moment, that is not very useful for our purposes. If one traces their proof, one can, conceivably, get explicit expressions for each specific moment, but they did not bother to implement it, and the asymptotics is not immediate.

We discovered, the following important fact.

Fact. Let $E_{1}(a, n):=E_{\text {area }}(a, n)$ be the expectation of the area statistic on $a$-parking functions of length $n$, given above, and let $E_{k}(n, a)$ be the $k$-th factorial moment

$$
E_{k}(n, a):=\frac{Q^{(k)}(n, a)(1)}{a(a+n)^{n-1}}
$$

then there exist polynomials $A_{k}(a, n)$ and $B_{k}(a, n)$ such that

$$
E_{k}(n, a)=A_{k}(a, n)+B_{k}(a, n) E_{1}(a, n)
$$

The beauty of experimental mathematics is that these can be found by cranking out enough data, using the sequence of probability generating functions $Q(n, a)(x)$, obtained by using the recurrence, (FundamentalRecurrenceAreaX), getting sufficiently many numerical data for the moments, and using undetermined coefficients. These can be proved a posteriori by taking these truly exact formulas and verifying that the implied recurrences for the $k$-th factorial moment (obtained from differentiating (FundamentalRecurrenceAreaX) $k$ times, using Leinitz's rule), in terms of the previous ones. But this is not necessary. Since, at the end of the day, it all boils down to verifying polynomial identities, so, once again, verifying them for sufficiently many different values of $(n, a)$ constitutes a rigorous proof. To be fully rigorous, one needs to prove a priori bounds for the degrees in $n$ and $a$, but, in our humble opinion, it is not that important, and could be left to the obtuse reader.

Our beloved computers, running the Maple package ParkingStatistics.txt, available from the front of this article

http://sites.math.rutgers.edu/〜zeilberg/mamarim/mamarimhtml/par.html, ,

produced the following, for the most interesting case of $a=1$, i.e. classical parking functions.

Theorem 1. (equivalent to a result in $[\mathrm{KY} 1]$ ): The expectation of the area statistic on parking functions of length $n$ is

$$
E_{1}(n):=-\frac{n}{2}+\frac{1}{2} \frac{(n+1) !}{(n+1)^{n}} \sum_{k=0}^{n-1} \frac{(n+1)^{k}}{k !}
$$

and asymptotically it equals $\frac{\sqrt{2 \pi}}{4} \cdot n^{3 / 2}+O(n)$. 
Theorem 2. The second factorial moment of the area statistic on parking functions of length $n$ is

$$
-\frac{7}{3}(n+1) E_{1}(n)+\frac{5}{12} n^{3}-\frac{1}{12} n^{2}-\frac{1}{3} n
$$

and asymptotically it equals $\frac{5}{12} \cdot n^{3}+O\left(n^{5 / 2}\right)$.

Theorem 3. The third factorial moment of the area statistic on parking functions of length $n$ is

$$
-\frac{175}{192} n^{4}-\frac{283}{192} n^{3}+\frac{199}{192} n^{2}+\frac{259}{192} n+\left(\frac{15}{32} n^{3}+\frac{521}{96} n^{2}+\frac{1219}{96} n+\frac{743}{96}\right) E_{1}(n),
$$

and asymptotically it equals $\frac{15}{128} \sqrt{2 \pi} \cdot n^{9 / 2}+O\left(n^{4}\right)$.

Theorem 4. The fourth factorial moment of the area statistic on parking functions of length $n$ is

$$
\begin{aligned}
& \frac{221}{1008} n^{6}+\frac{63737}{30240} n^{5}+\frac{101897}{15120} n^{4}+\frac{22217}{5040} n^{3}-\frac{1375}{189} n^{2}-\frac{187463}{30240} n \\
& +\left(-\frac{35}{16} n^{4}-\frac{449}{27} n^{3}-\frac{130243}{2520} n^{2}-\frac{7409}{105} n-\frac{503803}{15120}\right) E_{1}(n)
\end{aligned}
$$

and asymptotically it equals $\frac{221}{1008} \cdot n^{6}+O\left(n^{11 / 2}\right)$.

Theorem 5. The fifth factorial moment of the area statistic on parking functions of length $n$ is

$$
\begin{aligned}
& -\frac{105845}{110592} n^{7}-\frac{2170159}{290304} n^{6}-\frac{99955651}{3870720} n^{5}-\frac{30773609}{725760} n^{4}-\frac{94846903}{11612160} n^{3}+\frac{24676991}{483840} n^{2}+\frac{392763901}{11612160} n \\
& +\left(\frac{565}{2048} n^{6}+\frac{1005}{128} n^{5}+\frac{9832585}{165888} n^{4}+\frac{1111349}{5184} n^{3}+\frac{826358527}{1935360} n^{2}+\frac{159943787}{362880} n+\frac{1024580441}{5806080}\right) E_{1}(n)
\end{aligned}
$$

and asymptotically it equals $\frac{565}{8192} \sqrt{2 \pi} \cdot n^{15 / 2}+O\left(n^{7}\right)$.

Theorem 6. The sixth factorial moment of the area statistic parking functions of length $n$ is

$$
\begin{gathered}
\frac{82825}{576576} n^{9}+\frac{373340075}{110702592} n^{8}+\frac{9401544029}{332107776} n^{7}+\frac{14473244813}{127733760} n^{6}+\frac{414139396709}{1660538880} n^{5} \\
+\frac{88215445651}{332107776} n^{4}-\frac{18783816473}{332107776} n^{3}-\frac{643359542029}{1660538880} n^{2}-\frac{358936540409}{1660538880} n \\
+\left(-\frac{3955}{2048} n^{7}-\frac{186349}{6144} n^{6}-\frac{259283273}{1161216} n^{5}-\frac{119912501}{129024} n^{4}-\frac{149860633081}{63866880} n^{3}\right. \\
\left.-\frac{601794266581}{166053888} n^{2}-\frac{864000570107}{276756480} n-\frac{921390308389}{830269440}\right) E_{1}(n),
\end{gathered}
$$

and asymptotically it equals $\frac{82825}{576576} \cdot n^{9}+O\left(n^{17 / 2}\right)$.

For Theorems 7-30, see the output file

http://sites.math.rutgers.edu/ zeilberg/tokhniot/oParkingStatistics7.txt . 
Let $\left\{e_{k}\right\}_{k=1}^{\infty}$ be the sequence of moments of the Airy distribution, defined by the recurrence given in Equations (4) and (5) in Svante Janson's interesting survey paper [J]. Our computers, using our Maple package, proved that

$$
E_{k}(n)=e_{k} n^{\frac{3 k}{2}}+O\left(n^{\frac{3 k-1}{2}}\right)
$$

for $1 \leq k \leq 30$. It follows that the limiting distribution of the area statistic is (most probably) the Airy distribution, since the first 30 moments match. Of course, this was already known to continuous probability theorists, and we only proved it for the first 30 moments, but:

- Our methods are purely elementary and finitistic

- We can easily go much farther, i.e. prove it for more moments

- We believe that our approach, using recurrences, can be used to derive a recurrence for the leading asymptotics of the factorial moments, $E_{k}(n)$, that would turn out to be the same as the above mentioned recurrence (Eqs. (4) and (5) in $[\mathrm{J}]$ ). We leave this as a challenge to the reader.

Exact expressions for the first 10 moments of the Area statistic for general $a$-parking

To see expressions in $a, n$, and $E_{1}(n, a)$, for the first 10 moments of $a$-parking, see

http://sites.math.rutgers.edu/ ${ }^{\sim}$ zeilberg/tokhniot/oParkingStatistics8.txt .

Acknowledgment: Many thanks are due to Valentin Féray and Svante Janson for insightful information and useful references. Also thanks to Benji Weiss for comments on a previous version.

\section{References}

[DH] Persi Diaconis and Angela Hicks, Probabilizing Parking Functions, Adv. in Appl. Math. 89 (2017), 125-155.

https://arxiv.org/abs/1611.09821.

[EZ] Shalosh B. Ekhad and Doron Zeilberger, Going Back to Neil Sloane's FIRST LOVE (OEIS Sequence A435): On the Total Heights in Rooted Labeled Trees, The Personal Journal of Shalosh B. Ekhad and Doron Zeilberger, July 19, 2016.

http://sites.math.rutgers.edu/ ${ }^{\sim}$ zeilberg/mamarim/mamarimhtml/a435.html (accessed May 24, 2018) .

[FR] Dominique Foata and John Riordan, Mapping of acyclic and parking functions, Aequationes Mathematicae 10 (1974), 490-515.

[J] Svante Janson, Brownian excursion area, Wrights constants in graph enumeration, and other Brownian areas, Probab. Surveys, 4(2007), 80-145.

https://projecteuclid.org/euclid.ps/1178804352 (accessed May 24, 2018) .

[KW] Alan G. Konheim and Benjamin Weiss, An occupancy discipline and applications, SIAM J. 
Applied Math. 14 (1966), 1266-1274. [Available from JSTOR.]

[KY1] J.P. Kung and C. Yan, Expected sums of general parking functions, Annals of Combinatorics 7 (2003), 481-493.

[KY2] J.P. Kung and C. Yan, Exact formulas for the moments of sums of classical parking functions, Advances in Applied Mathematics 31 (2003), 215-241.

[RS] John Riordan and Neil J. A. Sloane, The enumeration of rooted trees by total height, J. Australian Math. Soc. 10 (1969), 278-282.

http://neilsloane.com/doc/riordan-enum-trees-by-height.pdf (accessed May 24, 2018) .

[Sc] Marcel-Paul Schützenberger, On an enumeration problem, J. Combinatorial Theory 4 (1968), 219-221.

[St1] Richard Stanley, Parking functions,

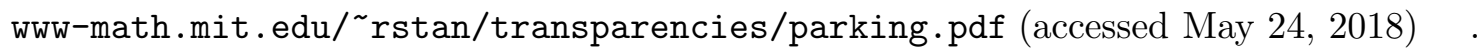

[St2] Richard Stanley, A survey of parking functions,

www-math.mit.edu/ rstan/transparencies/parking3.pdf (accessed May 24, 2018) .

[St3] Richard Stanley, "Enumerative Combinatorics, Volume 2", Cambridge University Press, 1999.

[W] Herbert S. Wilf, What is an Answer?, The American Mathematical Monthly 89 (1982), 289292.

[Z1] Doron Zeilberger, Symbolic Moment Calculus I.: Foundations and Permutation Pattern Statistics, Annals of Combinatorics 8 (2004), 369-378.

http://sites.math.rutgers.edu/ ${ }^{\sim}$ zeilberg/mamarim/mamarimhtml/smcI.html (accessed May $24,2018)$.

[Z2] Doron Zeilberger, Symbolic Moment Calculus II.: Why is Ramsey Theory Sooooo Eeeenormously Hard?, INTEGERS 7(2)(2007), A34.

http://sites.math.rutgers.edu/ zeilberg/mamarim/mamarimhtml/smcII.html (accessed May $24,2018)$.

[Z3] Doron Zeilberger, The $n^{n-2}$-th proof for the number of labeled trees, The Personal Journal of Shalosh B. Ekhad and Doron Zeilberger, undated (c. 1998), http://sites.math.rutgers.edu/ ${ }^{\sim}$ zeilberg/mamarim/mamarimhtml/labtree.html (accessed May 24, 2018).

[Z4] Doron Zeilberger, The Automatic Central Limit Theorems Generator (and Much More!), "Advances in Combinatorial Mathematics: Proceedings of the Waterloo Workshop in Computer Algebra 2008 in honor of Georgy P. Egorychev", chapter 8, pp. 165-174, (I.Kotsireas, E.Zima, eds. Springer Verlag, 2009.) 
http://sites.math.rutgers.edu/ zeilberg/mamarim/mamarimhtml/georgy.html (accessed May 24, 2018)

Yukun Yao, Department of Mathematics, Rutgers University (New Brunswick), Hill Center-Busch Campus, 110 Frelinghuysen Rd., Piscataway, NJ 08854-8019, USA.

Email: yao at math dot rutgers dot edu.

Doron Zeilberger, Department of Mathematics, Rutgers University (New Brunswick), Hill CenterBusch Campus, 110 Frelinghuysen Rd., Piscataway, NJ 08854-8019, USA.

Email: DoronZeil at gmail dot com .

First Written: June 5, 2018 ; This version: June 6, 2018. 